\title{
Analysis and Design of a CMOS Common- Source Cross Coupled Amplifier with NMOS only Active Inductor
}

\author{
Kittipong Tripetch, Parkpoom Khanthawiworn \\ Division of Electronic and Telecommunication Engineering \\ Faculty of Engineering and Architecture, Rajamangala University of Technology Suvarnabhumi \\ Nonthaburi, Thailand \\ Received: April 25, 2021. Received: October 5, 2021. Accepted: October 26, 2021. Published: November 5, 2021.
}

\begin{abstract}
The polynomial form of the input impedance of a CMOS cross-coupled amplifier with NMOS only active inductor are proposed. The formula of polynomial form and novel coefficients are programmed in Scilab and MATLAB so that the pole position of the fourth order polynomial can be found. From experience of a simple cross coupled oscillator design, the proposed circuit diagram may not oscillate as a triangular wave signal because the two poles are complex (underdamped case) but its real part could not be minimized to 0.001 so that it has a stable amplitude and additional two poles are real poles. The impulse sensitivity function (ISF) of the proposed circuit is derived for the first time so that it may be used to derive phase noise in the future.
\end{abstract}

Keywords_bandpass amplifier, bandpass filter, oscillator.

\section{INTRODUCTION}

$\mathrm{A}$ Simple cross-coupled oscillator and a regulated crosscoupled oscillator are proposed and designed long time ago [1]-[4]. A simple cross coupled oscillator input impedance was derived to have a fourth order polynomial form. But the proposed circuit has six transistors compared with two transistors of a simple cross-coupled oscillator with passive resonance circuit. The proposed circuit do not use passive resonance circuit compared with a simple cross coupled oscillator and a regulated cascode cross coupled oscillator. As a result, it may have a higher order polynomial form of input impedance because it use more transistors. The advantage of a simple cross coupled oscillator is that it is derived to oscillate as a triangular waveform which is plotted with MATLAB but the disadvantage is that its triangular wave signal fluctuate between dc supple voltage. This paper is used to describe how to analyze and design a common source cross-coupled amplifier with NMOS only active inductor with the hope that it may be used as a higher frequency oscillator compared with a simple cross coupled oscillator.
Section II describes the description of the circuit and a derivation of the input impedance of the proposed circuit

Section III describes how does the phase noise analysis of the proposed circuit diagram by impulse sensitivity function (ISF) could be derived.

Section IV describes how to design and simulate with MATLAB the pole position and how it is moved in the complex plane plot by varying the small signal parameters as a function of drain current and passive capacitor.

.The active inductor may be published for the first time by Hara [5]-[6]. They do not use the concept of ground inductor and floating active inductor which used a single transistor as a transconductance amplifier connect output as an input of another transconductance amplifier. In addition, the active inductor which use this concept have some advantage. It can have an input impedance as a resonance bandpass filter.

The negative impedance converter (NIC) can be used as a gyrator for a video frequency filter [7]. But this type of gyrator can be seen as a ground inductor. The floating positive and negative inductor can be designed with a large inductance values greater than $2 \mathrm{nH}$ which was published since 1995 [8]

The quality factor of the bandpass filter can be enhanced by Q enhanced technique which can tune quality factor from 10490. This technique was published since 1997 [9]. The gyrator can be implemented by transmission line which can be used in power processing circuits [10]. The additional Q enhanced technique was proposed by Yodprasit [11]. It is called double feedback and cascode double feedback where there are two feedback loops from the input to the point of feedback which look like common-gate for the drain input terminal. For the first loop, the signal propagates by common-source and common-drain connection. For the second loop, the signal propagates by common-gate, common-gate and common-drain connection. This technique can boost quality factor to 12,000 .

The common-gate cascade with common-source was proposed as an active inductor core circuit for bandpass amplifier since 2001[12]. The two transistor of active inductor was proposed 8 circuit diagrams as the core of bandpass 
amplifier which was published since 2002 [13].

\section{THE DESCRIPTION OF THE CIRCUIT AND A DERIVATION OF INPUT IMPEDANCE OF THE PROPOSED CIRCUIT}

\section{A. The description of the circuit}

The proposed circuit is called "A common-source cross coupled amplifier with NMOS only active inductor. It is shown in figurel. The circuit composed of 6 NMOS transistors. The transistors M1, M2 are formed by a well known gyrator loop which composed of two transconductance amplifier which connected output of the transconductance amplifier with the input of other transconductance amplifier. It have a capacitive load at the output of the first transconductance amplifier. The transistor M4, M5 are connected as a second gyrator loop similar with the first gyrator loop. The passive circuit and bias circuit composed of two capacitors $(\mathrm{C} 1, \mathrm{C} 2)$ and two resistors $(\mathrm{R} 1, \mathrm{R} 2)$. It must have the capacitors for a degree of freedom in designing the active inductors. It must have a resistors to bias the transistor M2 and M5 so that it will be operating in a saturation region. The dashed line are drawn outside an active inductor to indicate the resonance circuit block diagram which can be replace with various types of active inductors as a sub-circuit of the common-source cross-coupled amplifier.

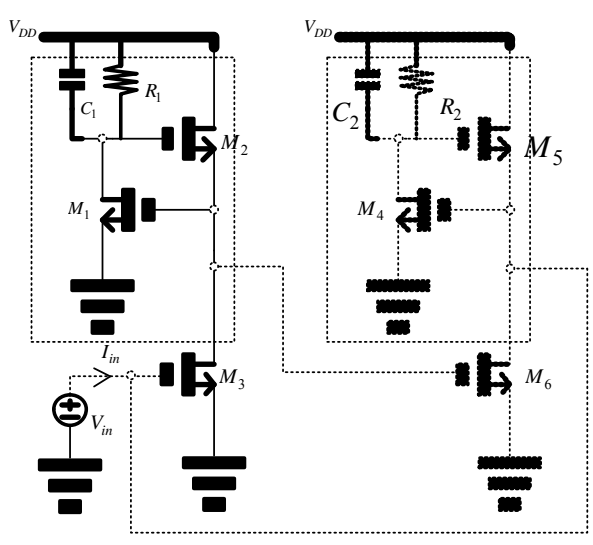

Figure 1. The Circuit diagram of "The common-source cross-coupled amplifier with NMOS only active inductor"

\section{B. The derivation of the input impedance of the common-} source cross-coupled amplifier with active inductor

The derivation of the input impedance of the proposed amplifier can be separated into three parts. Part1 is used to illustrate how to derive the input impedance of the active inductor which is connected as the load of the first stage amplifier which is shown in figure2. Part2 is used to illustrate how to derive the input impedance of the active inductor which is connected as the load of the second stage amplifier which is shown in figure3.Part3 is the used to illustrate the whole circuit which is shown in figure4.
The active inductor input impedance calculation methodology diagram is shown in the left hand side of figure2. The high frequency equivalent circuit of the NMOS only active inductor is shown in the right hand side of figure2.

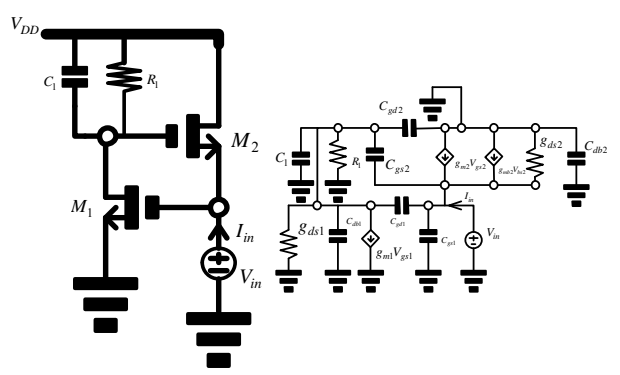

Figure 2. The input impedance of the NMOS only active inductor and its high frequency equivalent circuit of the first stage resonance circuit

$$
\begin{aligned}
& Z_{i n 1}=\frac{V_{i n}}{I_{i n}}=\frac{\left(s a_{4}+a_{5}\right)}{\left(s^{2} a_{7}+s a_{8}+a_{9}\right)}=\frac{s a_{4}+a_{5}}{s^{2}+\left(2 \omega_{n} \zeta\right) s+\omega_{n}^{2}} \\
& a_{1}=C_{g s 2}+C_{g d 1} \\
& a_{2}=C_{g s 2}+C_{d b 2}+C_{g d 1}+C_{g s 1} \\
& a_{3}=\left(g_{m 2}+g_{m b 2}+g_{d s 2}\right) \\
& a_{4}=\left(C_{d b 1}+C_{1}+C_{g s 2}+C_{g d 2}+C_{g d 1}\right) \\
& a_{5}=g_{d s 1}+\left(\frac{1}{R_{1}}\right) \\
& a_{6}=C_{g d 1}+C_{g s 2} \\
& a_{7}=\left(a_{2} a_{4}-\left(a_{6} a_{1}\right)\right) \\
& a_{8}=\left(a_{2} a_{5}+a_{3} a_{4}-\left(a_{6} g_{m 2}-g_{m 1} a_{1}\right)\right) \\
& a_{9}=\left(a_{3} a_{5}+g_{m 1} g_{m 2}\right)
\end{aligned}
$$

$Z_{i n 1}$ is the input impedance of the transistor M1 and M2 which form the gyrator loop in figure3. The novel coefficients $a_{1}, a_{2}, a_{3}, a_{4}, a_{5}, a_{6}, a_{7}, a_{8}, a_{9}$ are defined with parasitic capacitances of the transistor M1 and M2 and passive elements such as $\mathrm{C} 1$ and $\mathrm{R} 1$.

It is useful to design the circuit to oscillate, its input impedance denominator polynomial should be designed to have a characteristic similar with undamped polynomial so that its roots will have imaginary pole frequency. It can be written as follows 


$$
\begin{aligned}
& Z_{i n 1}=\frac{V_{i n}}{I_{i n}}=\frac{\left(s \frac{a_{4}}{a_{7}}+\frac{a_{5}}{a_{7}}\right)}{\left(s^{2}+s\left(\frac{a_{8}}{a_{7}}\right)+\left(\frac{a_{9}}{a_{7}}\right)\right)}=\frac{s \frac{a_{4}}{a_{7}}+\frac{a_{5}}{a_{7}}}{s^{2}+\left(2 \omega_{n} \zeta\right) s+\omega_{n}^{2}} \\
& \omega_{n}=\sqrt{\frac{a_{9}}{a_{7}}} \rightarrow\left(2 \omega_{n} \zeta\right)=\left(\frac{a_{8}}{a_{7}}\right) \rightarrow \zeta=\frac{a_{8}}{a_{7}\left(2 \sqrt{\frac{a_{9}}{a_{7}}}\right)}=\frac{a_{8}}{2 \sqrt{a_{9} a_{7}}}=0
\end{aligned}
$$

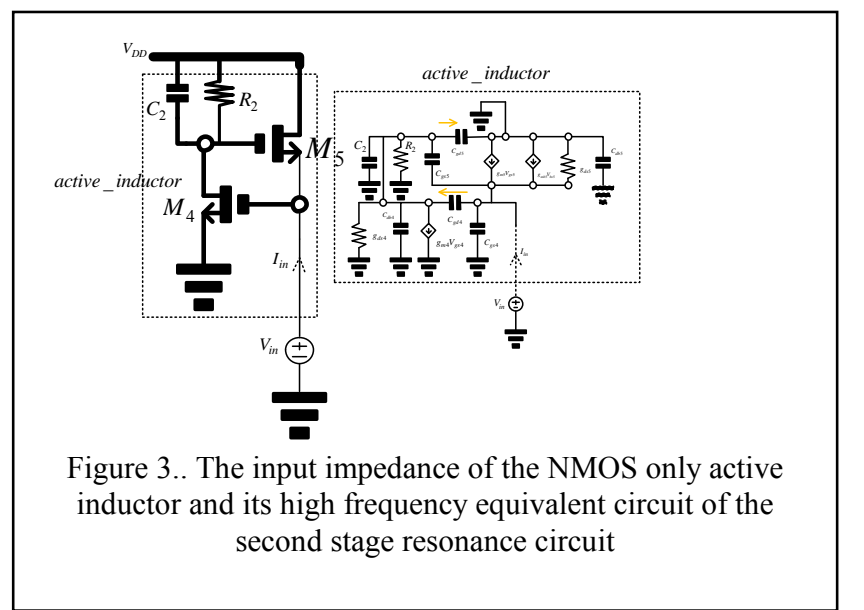

$Z_{\text {in } 2}=\frac{V_{\text {in } 2}}{I_{\text {in } 2}}=\frac{\left(s a_{13}+a_{14}\right)}{\left(s^{2} a_{16}+s a_{17}+a_{18}\right)}=\frac{s a_{13}+a_{14}}{s^{2}+\left(2 \omega_{n} \zeta\right) s+\omega_{n}^{2}}$

$a_{10}=C_{g s 5}+C_{g d 4}$

$a_{11}=C_{g s 5}+C_{g d 4}+C_{g s 4}$

$a_{12}=\left(g_{m 5}+g_{m b 5}+g_{d s 5}\right)$

$a_{13}=\left(C_{d b 4}+C_{2}+C_{g s 5}+C_{g d 5}+C_{g d 4}\right)$

$a_{14}=g_{d s 4}+\left(\frac{1}{R_{2}}\right)$

$a_{15}=C_{g d 4}+C_{g s 5}$

$a_{16}=\left(a_{11} a_{13}-\left(a_{15} a_{10}\right)\right)$

$a_{17}=\left(a_{11} a_{14}+a_{12} a_{13}-\left(a_{15} g_{m 5}-g_{m 4} a_{10}\right)\right)$

$a_{18}=\left(a_{12} a_{14}+g_{m 4} g_{m 5}\right)$

$Z_{\text {in } 2}$ is the input impedance of the transistor M4 and M5 which forms the gyrator loop in figure 4. The novel coefficients $a_{10}, a_{11}, a_{12}, a_{13}, a_{14}, a_{15}, a_{16}, a_{17}, a_{18}$ are defined with parasitic capacitances of the transistor M4, M5 and passive elements such as C2 and R2. The equations of input impedance of active inductor with NMOS only can be used to substitute as an impedance load of the common-source cross-coupled amplifier with NMOS only active inductor so that the derivation may be easier for analysis compared with directly analyze the high frequency equivalent circuit in figure 4 which is shown below.

Equation (1b) may not be designed to have equal value both sides of the equation easily. It is possible to add some passive capacitors in parallel with parasitic capacitances of the transistors so that an equality of equation (1b) is true.

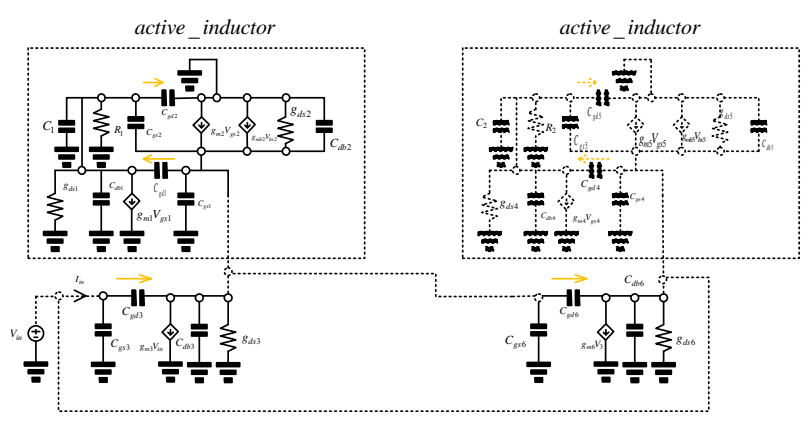

Figure 4. The high frequency equivalent circuit of the commonsource cross-coupled amplifier with NMOS only active inductor.

$$
\begin{aligned}
& b_{1}=C_{g d 6}+C_{g d 3}+C_{g s 3}+C_{d b 6} \\
& b_{2}=C_{g d 6}+C_{g d 3} \\
& b_{3}=C_{g d 3}+C_{g d 6} \\
& b_{4}=C_{g d 3}+C_{d b 3}+C_{g s 6}+C_{g d 6} \\
& b_{5}=\left(b_{4} a_{4}+a_{7}\right) \\
& b_{6}=\left(b_{4} a_{5}+g_{d s 3} a_{4}+a_{8}\right) \\
& b_{7}=\left(g_{d s 3} a_{5}+a_{9}\right) \\
& b_{8}=\left(b_{1} a_{13}+a_{16}\right) \\
& b_{9}=\left(b_{1} a_{14}+g_{d s 6} a_{13}+a_{17}\right) \\
& b_{10}=\left(g_{d s 6} a_{14}+a_{18}\right) \\
& b_{11}=\left(b_{3} a_{4}\right), b_{12}=\left(b_{3} a_{5}-g_{m 3} a_{4}\right), b_{13}=\left(g_{m 3} a_{5}\right) \\
& b_{14}=\left(b_{11} b_{2}\right), b_{15}=\left(b_{12} b_{2}-b_{11} g_{m 6}\right) \\
& b_{16}=\left(b_{12} g_{m 6}+b_{13} b_{2}\right), b_{17}=\left(b_{13} g_{m 6}\right)
\end{aligned}
$$

$$
Z_{\text {in }}=\left\lfloor\frac{\left(s^{3}\left(a_{13} b_{5}\right)+s^{2}\left(a_{13} b_{6}+a_{14} b_{5}\right)+s\left(a_{13} b_{7}+a_{14} b_{6}\right)+\left(a_{14} b_{7}\right)\right)}{\left(\begin{array}{l}
\left.s^{4}\left(b_{8} b_{5}-\left(a_{13} b_{14}\right)\right)+s^{3}\left(b_{8} b_{6}+b_{9} b_{5}-\left(a_{13} b_{15}+a_{14} b_{14}\right)\right)\right) \\
+s^{2}\left(b_{8} b_{7}+b_{9} b_{6}+b_{10} b_{5}-\left(a_{14} b_{15}-a_{13} b_{16}\right)\right) \\
+s\left(b_{9} b_{7}+b_{10} b_{6}-\left(a_{13} b_{17}-a_{14} b_{16}\right)\right)+\left(b_{10} b_{7}-\left(a_{14} b_{17}\right)\right)
\end{array}\right)}\right\rfloor
$$


$Z_{\text {in }}$ is an input impedance of the whole cross-couple amplifier with NMOS only active inductor. It is a polynomial form which can be used to design the amplifier and oscillator. The function inside numerator and denominator polynomial are defined with the novel coefficients in equation (1), (2) and (3).

The graph will be programmed with levell transistor model. The script can be written as a simple text file. The first section of program must define levell drain current model according to transistor model examples [4]. Drain current model are the function of electron mobility, oxide thickness, aspect ratio which are the width and length of the transistor MOSFET, threshold voltage, terminal voltage such as drain, gate, source terminal. Threshold voltage can be written as a function of initial threshold voltage, fermi potential and a source bulk voltage. The second section should define parasitic capacitances calculation. Parasitic capacitances of a high frequency equivalent circuit $\left(C_{g s}, C_{g d}, C_{d b}\right)$ is a function of

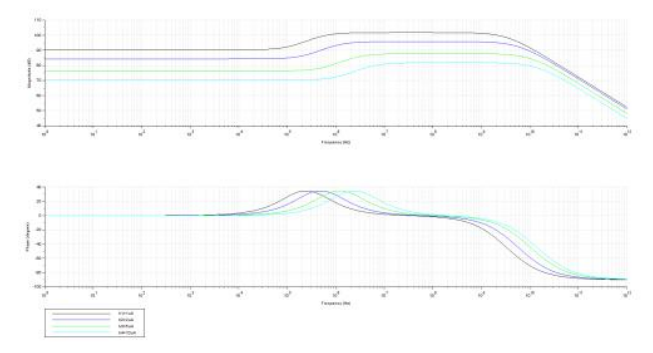

Figure 5. Input impedance of Magnitude and phase response as a function of current consumption

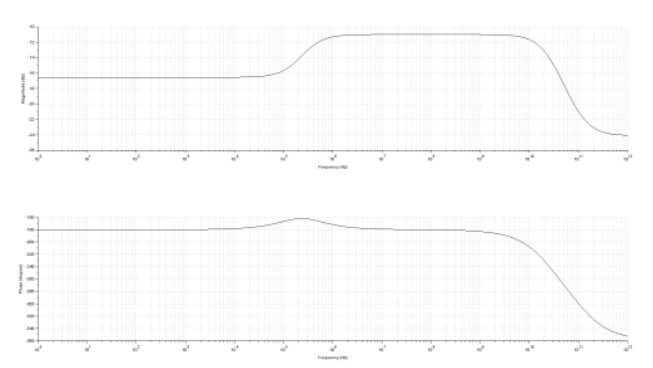

Figure 6. Magnitude and Phase response of the voltage transfer function without input current

In comparison with the reference [19], the coefficients of the polynomial which are listed in equation (3), (4) can be grouped and define with the novel coefficients name so that it is not duplicated with the coefficients in the equation (3),(4). It can be shown as following

$$
\begin{aligned}
& Z_{\text {in }}=\left[\frac{\left(s^{3} b_{18}+s^{2} b_{19}+s b_{20}+b_{21}\right)}{\left(s^{4} b_{22}+s^{3} b_{23}+s^{2} b_{24}+s b_{25}+b_{26}\right)}\right] \\
& b_{18}=\left(a_{13} b_{5}\right), b_{19}=\left(a_{13} b_{6}+a_{14} b_{5}\right), b_{20}=\left(a_{13} b_{7}+a_{14} b_{6}\right) \\
& b_{21}=\left(a_{14} b_{7}\right), b_{22}=\left(b_{8} b_{5}-\left(a_{13} b_{14}\right)\right) \\
& b_{23}=\left(b_{8} b_{6}+b_{9} b_{5}-\left(a_{13} b_{15}+a_{14} b_{14}\right)\right) \\
& b_{24}=\left(b_{8} b_{7}+b_{9} b_{6}+b_{10} b_{5}-\left(a_{14} b_{15}-a_{13} b_{16}\right)\right) \\
& b_{25}=\left(b_{9} b_{7}+b_{10} b_{6}-\left(a_{13} b_{17}-a_{14} b_{16}\right)\right) \\
& b_{26}=\left(b_{10} b_{7}-\left(a_{14} b_{17}\right)\right)
\end{aligned}
$$

From simulation results from reference [19]. The passive resonance circuit have some value which can make the circuit oscillate in MATLAB as a triangular waveform. From root locus as a function of current consumption. The polynomial form in equation (6) has some numerical coefficients which is borrowed from successful iterative simulation of MATLAB. These coefficients can be compared with the coefficients from equation (5). From this situation, someone may ask whether the coefficients $b_{22}, b_{23}, b_{24}, b_{25}, b_{26}$ can be designed to be equal with $b_{22}=d_{4}, b_{23}=d_{3}, b_{24}=d_{2}, b_{25}=d_{1}, b_{26}=1$

$$
\begin{aligned}
& Z_{i n}=\frac{s^{3}\left(L_{1} L_{2} C_{x 1}\right)+s^{2}\left(L_{1} L_{2}\left(g_{d s 2}+\frac{1}{R_{2}}\right)\right)+s\left(L_{1}\right)}{s^{4} d_{4}+s^{3} d_{3}+s^{2} d_{2}+s d_{1}+1} \\
& d_{4}=L_{1} C_{x 2} L_{2} C_{x 1}-L_{1} C_{2}\left(C_{g d 1}+C_{g d 2}\right)^{2} \\
& d_{3}=L_{1} C_{x 2} L_{2}\left(g_{d s 2}+\frac{1}{R_{2}}\right)+L_{1} L_{2} C_{x 1}\left(\frac{1}{R_{1}}+g_{d s 1}\right)+2 L_{1} L_{2} g_{m 2}\left(C_{g d 1}+C_{g d 2}\right) \\
& d_{2}=L_{1} C_{x 2}+L_{2} C_{x 1}+L_{1} L_{2}\left(g_{d s 1}+\frac{1}{R_{1}}\right)\left(g_{d s 2}+\frac{1}{R_{2}}\right)-L_{2}^{2} g_{m 2}^{2} \\
& d_{1}=L_{1}\left(\frac{1}{R_{1}}+g_{d s 1}\right)+L_{2}\left(\frac{1}{R_{2}}+g_{d s 2}\right)
\end{aligned}
$$

The fourth order general form partial fraction and the solution of inverse Laplace's transform can be written as following

$$
\begin{aligned}
& Z_{\text {in }}(s)=\frac{A_{1}}{s+f_{p 1}}+\frac{A_{2}}{s+f_{p 2}}+\frac{A_{3}}{s+f_{p 3}}+\frac{A_{4}}{s+f_{p 4}} \\
& z_{\text {in }}(t)=A_{1} e^{-f_{p 1} t}+A_{2} e^{-f_{p 2} t}+A_{3} e^{-f_{p 3} t}+A_{4} e^{-f_{p 4} t}
\end{aligned}
$$

From simulation results of the solutions which are related with level1 transistor model and small signal parameters of the MOSFET. The four poles system has two couple of complex conjugate poles. The pole relationships can be written as following. The first pole and second pole are complex conjugate pole. The third pole and fourth pole are complex conjugate pole. It can be written as following.

$$
f_{p 1}=f_{p 1 A}+j f_{p 1 B}, f_{p 2}=f_{p 1 A}-j f_{p 1 B}
$$




$$
f_{p 3}=f_{p 2 A}+j f_{p 2 B}, f_{p 4}=f_{p 2 A}-j f_{p 2 B}
$$

The fourth order unknown determination derivation solution is reference with [18]. It is derived to have a symbolic complex number.

$$
\begin{aligned}
& A_{1}=A_{11}+j A_{12} \\
& A_{2}=A_{21}+j A_{22} \\
& A_{3}=A_{31}+j A_{32} \\
& A_{4}=A_{41}+j A_{42}
\end{aligned}
$$

The complex conjugate poles in equation (8) and (9) and symbolic unknown in equation (10) can be replace with equation (7) as following

$$
\begin{aligned}
& z_{i n}(t)=A_{1} e^{-f_{p 1} t}+A_{2} e^{-f_{p 2} t}+A_{3} e^{-f_{p 3} t}+A_{4} e^{-f_{p 4} t} \\
& z_{i n}(t)=\left(A_{11}+j A_{12}\right) e^{-\left(f_{p 1 A}+j f_{p 1 B}\right) t}+\left(A_{21}+j A_{22}\right) e^{-\left(f_{p 1 A}-j f_{p 1 B}\right) t} \\
& +\left(A_{31}+j A_{32}\right) e^{-\left(f_{p 2 A}+j f_{p 2 B}\right) t}+\left(A_{41}+j A_{42}\right) e^{-\left(f_{p 2 A}-j f_{p 2 B}\right) t} \\
& \quad z_{i n}(t)=\left(A_{11}+j A_{12}\right) e^{-\left(f_{p 1 A}\right) t}\left[\cos \left(f_{p 1 B} t\right)-j \sin \left(f_{p 1 B} t\right)\right] \\
& +\left(A_{21}+j A_{22}\right) e^{-\left(f_{p 1 A}\right) t}\left[\cos \left(f_{p 1 B} t\right)+j \sin \left(f_{p 1 B} t\right)\right] \\
& +\left(A_{31}+j A_{32}\right) e^{-\left(f_{p 2 A}\right) t}\left[\cos \left(f_{p 2 B} t\right)-j \sin \left(f_{p 2 B} t\right)\right] \\
& +\left(A_{41}+j A_{42}\right) e^{-\left(f_{p 2 A}\right) t}\left[\cos \left(f_{p 2 B} t\right)+j \sin \left(f_{p 2 B} t\right)\right]
\end{aligned}
$$

\section{PHASE NOISE DERIVATION OF THE COMMON-SOURCE CROSS-COUPLED OSCILLATOR WITH ACTIVE INDUCTOR}

Since the impulse sensitivity function (ISF) is used to derived phase noise of some type of oscillator. The ISF may be used to derived from the Fourier series which is borrowed from the principle in reference [20]

$$
\Gamma\left(\omega_{0} \tau\right)=c_{0}+\sum_{n=1}^{\infty} c_{n} \cos \left(n \omega_{0} \tau+\theta_{n}\right)
$$

The time domain function of the common-source crosscoupled oscillator with active inductor can be derived from inverse Laplace's transform of the partial fraction expansion general form which are composed of two steps for a derivation. The first step is unknown determination which can be derived from factorization form cross multiplication of problem to general form of partial fraction. The Fourier series and Fourier coefficient are borrowed from reference [21]

$$
\begin{aligned}
& f(t)=a_{0}+\sum_{n=1}^{\infty}\left(a_{n} \cos \left(n \omega_{0} t\right)+b_{n} \sin \left(n \omega_{0} t\right)\right) \\
& a_{0}=\frac{1}{T} \int_{0}^{T} f(t) d t=c_{0} \\
& a_{0}=\frac{1}{T} \int_{0}^{T}\left[\begin{array}{l}
\left(A_{11}+j A_{12}\right) e^{-\left(f_{p 1 A}+j f_{p 1 B}\right) t} \\
+\left(A_{21}+j A_{22}\right) e^{-\left(f_{p 1 A}-j f_{p 1 B}\right) t} \\
+\left(A_{31}+j A_{32}\right) e^{-\left(f_{p 2 A}+j f_{p 2 B}\right) t} \\
+\left(A_{41}+j A_{42}\right) e^{-\left(f_{p 2 A}-j f_{p 2 B}\right) t}
\end{array}\right] d t
\end{aligned}
$$

$z_{\text {in }}(t)=\left(A_{11} \cos \left(f_{p 1 B} t\right)+A_{12} \sin \left(f_{p 1 B} t\right)\right) e^{-\left(f_{p 1 A}\right) t}+j\left(A_{12} \cos \left(f_{p 1 B} t\right)-A_{11} \sin \left(f_{p 1 B} t\right)\right) e^{-\left(f_{p 11}\right) t}$

$+\left(A_{21} \cos \left(f_{p 1 B} t\right)-A_{22} \sin \left(f_{p 1 B} t\right)\right) e^{-\left(f_{p 14}\right) t}+j\left(A_{22} \cos \left(f_{p 1 B} t\right)+A_{21} \sin \left(f_{p 1 B} t\right)\right) e^{-\left(f_{p 1 A}\right) t}$ The complex amplitude and complex trigonometry function $\left.+\left(A_{31} \cos \left(f_{p 2 B} t\right)+A_{32} \sin \left(f_{p 2 B} t\right)\right) e^{-\left(f_{p 2 A}\right) t}+j\left(A_{32} \cos \left(f_{p 2 B} t\right)-A_{31} \sin \left(f_{p 2 B} t\right)\right) e^{- \text {tan }}\right) b$ integrated as following

$+\left(A_{41} \cos \left(f_{p 2 B} t\right)-A_{42} \sin \left(f_{p 2 B} t\right)\right) e^{-\left(f_{p 2 A}\right) t}+j\left(A_{42} \cos \left(f_{p 2 B} t\right)+A_{41} \sin \left(f_{p 2 B} t\right)\right) e^{-\left(f_{p 2 A}\right) t}$

The input impedance can be grouped with real parts which are composed of 8 terms and imaginary parts which are composed of additional 8 terms.

$$
\begin{aligned}
& z_{i n}(t)=\left(\begin{array}{l}
A_{11} \cos \left(f_{p 1 B} t\right)+A_{12} \sin \left(f_{p 1 B} t\right) \\
+A_{21} \cos \left(f_{p 1 B} t\right)-A_{22} \sin \left(f_{p 1 B} t\right)
\end{array}\right) e^{-\left(f_{p 1 A}\right) t}+j\left(\begin{array}{l}
A_{12} \cos \left(f_{p 1 B} t\right)-A_{11} \sin \left(f_{p 1 B} t\right) \\
+A_{22} \cos \left(f_{p 1 B} t\right)+A_{21} \sin \left(f_{p 1 B} t\right)
\end{array}\right) e^{-\left(f_{p 1 A}\right) t} \\
& +\left(\begin{array}{l}
A_{31} \cos \left(f_{p 2 B} t\right)+A_{32} \sin \left(f_{p 2 B} t\right) \\
+A_{41} \cos \left(f_{p 2 B} t\right)-A_{42} \sin \left(f_{p 2 B} t\right)
\end{array}\right) e^{-\left(f_{p 2 A}\right) t}+j\left(\begin{array}{l}
A_{32} \cos \left(f_{p 2 B} t\right)-A_{31} \sin \left(f_{p 2 B} t\right) \\
+A_{42} \cos \left(f_{p 2 B} t\right)+A_{41} \sin \left(f_{p 2 B} t\right)
\end{array}\right) e^{-\left(f_{p 2 A}\right) t}
\end{aligned}
$$

$$
a_{0}=-\frac{1}{T}\left[\begin{array}{l}
\frac{\left(A_{11}+j A_{12}\right)}{\left(f_{p 1 A}+j f_{p 1 B}\right)} e^{-\left(f_{p 1 A}+j f_{p 1 B}\right) t} \\
+\frac{\left(A_{21}+j A_{22}\right)}{\left(f_{p 1 A}-j f_{p 1 B}\right)} e^{-\left(f_{p 1 A}-j f_{p 1 B}\right) t} \\
+\frac{\left(A_{31}+j A_{32}\right)}{\left(f_{p 2 A}+j f_{p 2 B}\right)} e^{-\left(f_{p 2 A}+j f_{p 2 B}\right) t} \\
+\frac{\left(A_{41}+j A_{42}\right)}{\left(f_{p 2 A}-j f_{p 2 B}\right)} e^{-\left(f_{p 2 A}-j f_{p 2 B}\right) t}
\end{array}\right]_{0}^{T}
$$

After take integration limit in equation (18), it has a result as follows 


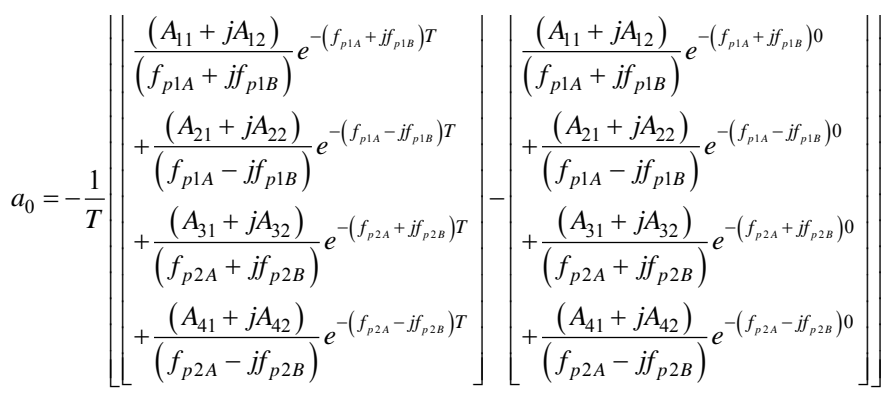

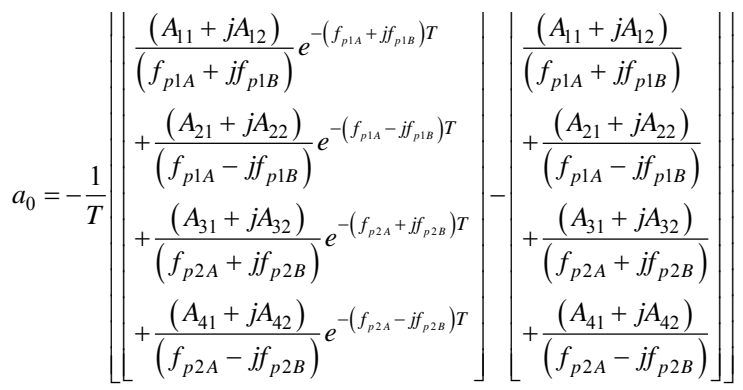

$$
\begin{aligned}
& a_{n}=\frac{2}{T} \int_{0}^{T} f(t) \cos \left(n \omega_{0} t\right) d t \\
& a_{n}=\frac{2}{T} \int_{0}^{T}\left[\begin{array}{l}
\left(A_{11}+j A_{12}\right) e^{-\left(f_{p 1 A}+j f_{p 1 B}\right) t} \\
+\left(A_{21}+j A_{22}\right) e^{-\left(f_{p 1 A}-j f_{p 1 B}\right) t} \\
+\left(A_{31}+j A_{32}\right) e^{-\left(f_{p 2 A}+j f_{p 2 B}\right) t} \\
+\left(A_{41}+j A_{42}\right) e^{-\left(f_{p 2 A}-j f_{p 2 B}\right) t}
\end{array}\right]\left[\frac{e^{j n \omega_{0} t}+e^{-j n \omega_{0} t}}{2}\right] d t
\end{aligned}
$$

The Fourier coefficient can be performing integration easily by separation of the exponential of positive and negative imaginary part as following.

$$
\begin{gathered}
a_{n}=\frac{2}{2 T} \int_{0}^{T}\left[\begin{array}{l}
\left(A_{11}+j A_{12}\right) e^{-\left(f_{p 1 A}+j f_{p 1 B}\right) t} e^{j n \omega_{0} t} \\
+\left(A_{21}+j A_{22}\right) e^{-\left(f_{p 1 A}-j f_{p 1 B}\right) t} e^{j n \omega_{0} t} \\
+\left(A_{31}+j A_{32}\right) e^{-\left(f_{p 2 A}+j f_{p 2 B}\right) t} e^{j n \omega_{0} t} \\
+\left(A_{41}+j A_{42}\right) e^{-\left(f_{p 2 A}-j f_{p 2 B}\right) t} e^{j n \omega_{0} t}
\end{array}\right] d t \\
+\frac{2}{2 T} \int_{0}^{T}\left[\begin{array}{l}
\left(A_{11}+j A_{12}\right) e^{-\left(f_{p 1 A}+j f_{p 1 B}\right) t} e^{-j n \omega_{0} t} \\
+\left(A_{21}+j A_{22}\right) e^{-\left(f_{p 1 A}-j f_{p 1 B}\right) t} e^{-j n \omega_{0} t} \\
+\left(A_{31}+j A_{32}\right) e^{-\left(f_{p 2 A}+j f_{p 2 B}\right) t} e^{-j n \omega_{0} t} \\
+\left(A_{41}+j A_{42}\right) e^{-\left(f_{p 2 A}-j f_{p 2 B}\right) t} e^{-j n \omega_{0} t}
\end{array}\right] d t
\end{gathered}
$$

The Fourier coefficient can be performing integration easily by grouping of the positive and negative exponential of imaginary frequency as follows

$$
\begin{gathered}
a_{n}=\frac{1}{T} \int_{0}^{T}\left[\begin{array}{l}
\left(A_{11}+j A_{12}\right) e^{-\left(f_{p 1 A}+j\left(f_{p 1 B}-n \omega_{0}\right)\right) t} \\
+\left(A_{21}+j A_{22}\right) e^{-\left(f_{p 1 A}-j\left(f_{p 1 B}+n \omega_{0}\right)\right) t} \\
+\left(A_{31}+j A_{32}\right) e^{-\left(f_{p 2 A}+j\left(f_{p 2 B}-n \omega_{0}\right)\right) t} \\
+\left(A_{41}+j A_{42}\right) e^{-\left(f_{p 2 A}-j\left(f_{p 2 B}+n \omega_{0}\right)\right) t}
\end{array}\right] d t \\
+\frac{1}{T} \int_{0}^{T}\left[\begin{array}{l}
\left(A_{11}+j A_{12}\right) e^{-\left(f_{p 1 A}+j\left(f_{p 1 B}+n \omega_{0}\right)\right) t} \\
+\left(A_{21}+j A_{22}\right) e^{-\left(f_{p 1 A}-j\left(f_{p 1 B}-n \omega_{0}\right)\right) t} \\
+\left(A_{31}+j A_{32}\right) e^{-\left(f_{p 2 A}+j\left(f_{p 2 B}+n \omega_{0}\right)\right) t} \\
+\left(A_{41}+j A_{42}\right) e^{-\left(f_{p 2 A}-j\left(f_{p 2 B}-n \omega_{0}\right)\right) t}
\end{array}\right] d t
\end{gathered}
$$

The integration of exponential of complex frequency can be performed with equation (20c). Then, the limit of integration (which are the period and zero) can be seen at the right hand side of the bracket.

$$
\begin{aligned}
& a_{n}=\frac{1}{T}\left[\begin{array}{l}
\frac{\left(A_{11}+j A_{12}\right)}{-\left(f_{p 1 A}+j\left(f_{p 1 B}-n \omega_{0}\right)\right)} e^{-\left(f_{p 1 A}+j\left(f_{p 1 B}-n \omega_{0}\right)\right) t} \\
-\frac{\left(A_{21}+j A_{22}\right)}{-\left(f_{p 1 A}-j\left(f_{p 1 B}+n \omega_{0}\right)\right)} e^{-\left(f_{p 1 A}-j\left(f_{p 1 B}+n \omega_{0}\right)\right) t} \\
+\frac{\left(A_{31}+j A_{32}\right)}{-\left(f_{p 2 A}+j\left(f_{p 2 B}-n \omega_{0}\right)\right)} e^{-\left(f_{p 2 A}+j\left(f_{p 2 B}-n \omega_{0}\right)\right) t} \\
+\frac{\left(A_{41}+j A_{42}\right)}{-\left(f_{p 2 A}-j\left(f_{p 2 B}+n \omega_{0}\right)\right)} e^{-\left(f_{p 2 A}-j\left(f_{p 2 B}+n \omega_{0}\right)\right) t}
\end{array}\right]_{0}^{T} \\
& +\frac{1}{T}\left[\begin{array}{l}
\frac{\left(A_{11}+j A_{12}\right)}{-\left(f_{p 1 A}+j\left(f_{p 1 B}+n \omega_{0}\right)\right)} e^{-\left(f_{p 1 A}+j\left(f_{p 1 B}+n \omega_{0}\right)\right) t} \\
+\frac{\left(A_{21}+j A_{22}\right)}{-\left(f_{p 1 A}-j\left(f_{p 1 B}-n \omega_{0}\right)\right)} e^{-\left(f_{p 1 A}-j\left(f_{p 1 B}-n \omega_{0}\right)\right) t} \\
+\frac{\left(A_{31}+j A_{32}\right)}{-\left(f_{p 2 A}+j\left(f_{p 2 B}+n \omega_{0}\right)\right)} e^{-\left(f_{p 2 A}+j\left(f_{p 2 B}+n \omega_{0}\right)\right) t} \\
+\frac{\left(A_{41}+j A_{42}\right)}{-\left(f_{p 2 A}-j\left(f_{p 2 B}-n \omega_{0}\right)\right)} e^{-\left(f_{p 2 A}-j\left(f_{p 2 B}-n \omega_{0}\right)\right) t}
\end{array}\right]_{0}^{T}
\end{aligned}
$$

The minus sign is the result of integration of exponential of complex frequency as a function of time. This minus sign can be move out of the bracket. Then, the limit of integration can be substitute into the time variable as follows 


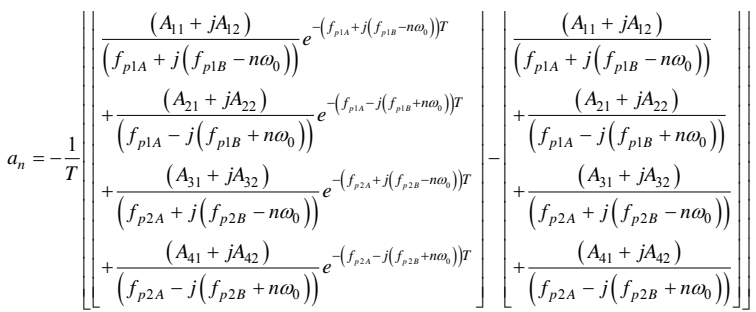

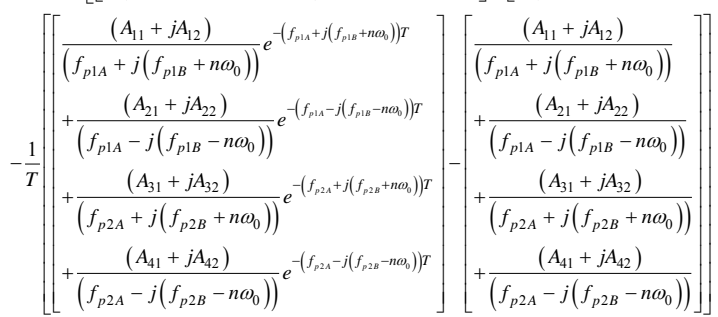

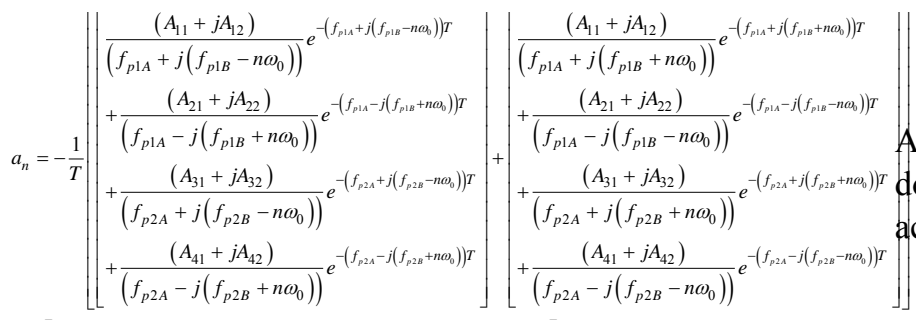

$$
\left.+\frac{1}{T}\left[\begin{array}{l}
\frac{\left(A_{11}+j A_{12}\right)}{\left(f_{p 1 A}+j\left(f_{p 1 B}-n \omega_{0}\right)\right)} \\
+\frac{\left(A_{21}+j A_{22}\right)}{\left(f_{p 1 A}-j\left(f_{p 1 B}+n \omega_{0}\right)\right)} \\
+\frac{\left(A_{31}+j A_{32}\right)}{\left(f_{p 2 A}+j\left(f_{p 2 B}-n \omega_{0}\right)\right)} \\
+\frac{\left(A_{41}+j A_{42}\right)}{\left(f_{p 2 A}-j\left(f_{p 2 B}+n \omega_{0}\right)\right)}
\end{array}\right]+\left[\begin{array}{c}
\frac{\left(A_{11}+j A_{12}\right)}{\left(f_{p 1 A}+j\left(f_{p 1 B}+n \omega_{0}\right)\right)} \\
+\frac{\left(A_{21}+j A_{22}\right)}{\left(f_{p 1 A}-j\left(f_{p 1 B}-n \omega_{0}\right)\right)} \\
+\frac{\left(A_{31}+j A_{32}\right)}{\left(f_{p 2 A}+j\left(f_{p 2 B}+n \omega_{0}\right)\right)} \\
+\frac{\left(A_{41}+j A_{42}\right)}{\left(f_{p 2 A}-j\left(f_{p 2 B}-n \omega_{0}\right)\right)}
\end{array}\right]\right]
$$

(20f)

It is necessary to multiply each denominator with its complex conjugation to each term in equation (20f). As a result, it can be written as follow

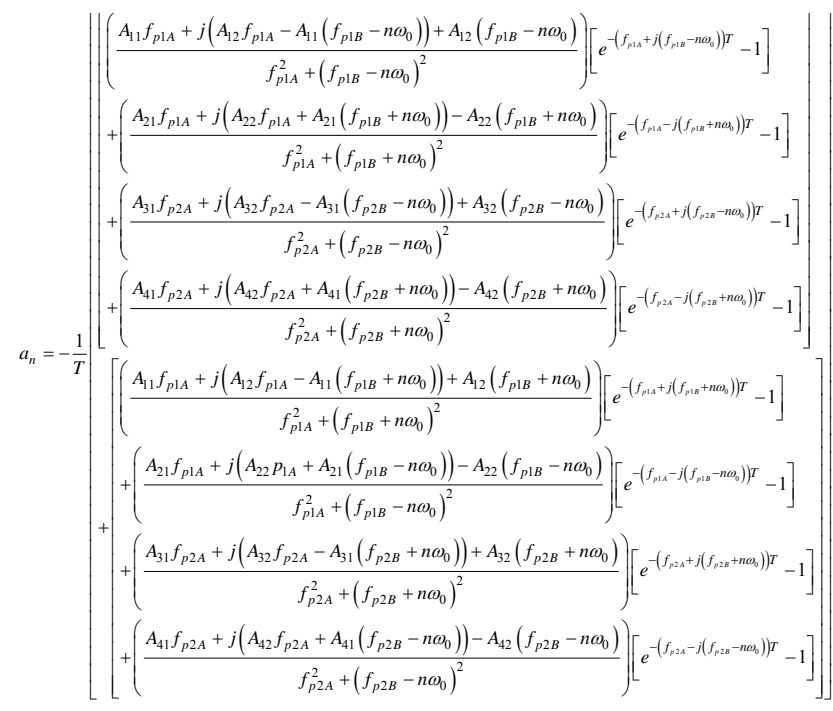

(20h)

Another Fourier coefficient formula can be substitute by time domain function of a common-source cross coupled with active inductor as follows

$$
\begin{aligned}
& b_{n}=\frac{2}{T} \int_{0}^{T} f(t) \sin \left(n \omega_{0} t\right) d t \\
& b_{n}=\frac{2}{T} \int_{0}^{T}\left[\begin{array}{l}
\left(A_{11}+j A_{12}\right) e^{-\left(f_{p 1 A}+j f_{p 1 B}\right) t} \\
+\left(A_{21}+j A_{22}\right) e^{-\left(f_{p 1 A}-j f_{p 1 B}\right) t} \\
+\left(A_{31}+j A_{32}\right) e^{-\left(f_{p 2 A}+j f_{p 2 B}\right) t} \\
+\left(A_{41}+j A_{42}\right) e^{-\left(f_{p 2 A}-j f_{p 2 B}\right) t}
\end{array}\right]\left[\frac{e^{j n \omega_{0} t}-e^{-j n \omega_{0} t}}{2 j}\right] d t
\end{aligned}
$$

The Fourier coefficient can be performing integration easily by separation of the exponential of positive and negative imaginary part as following.

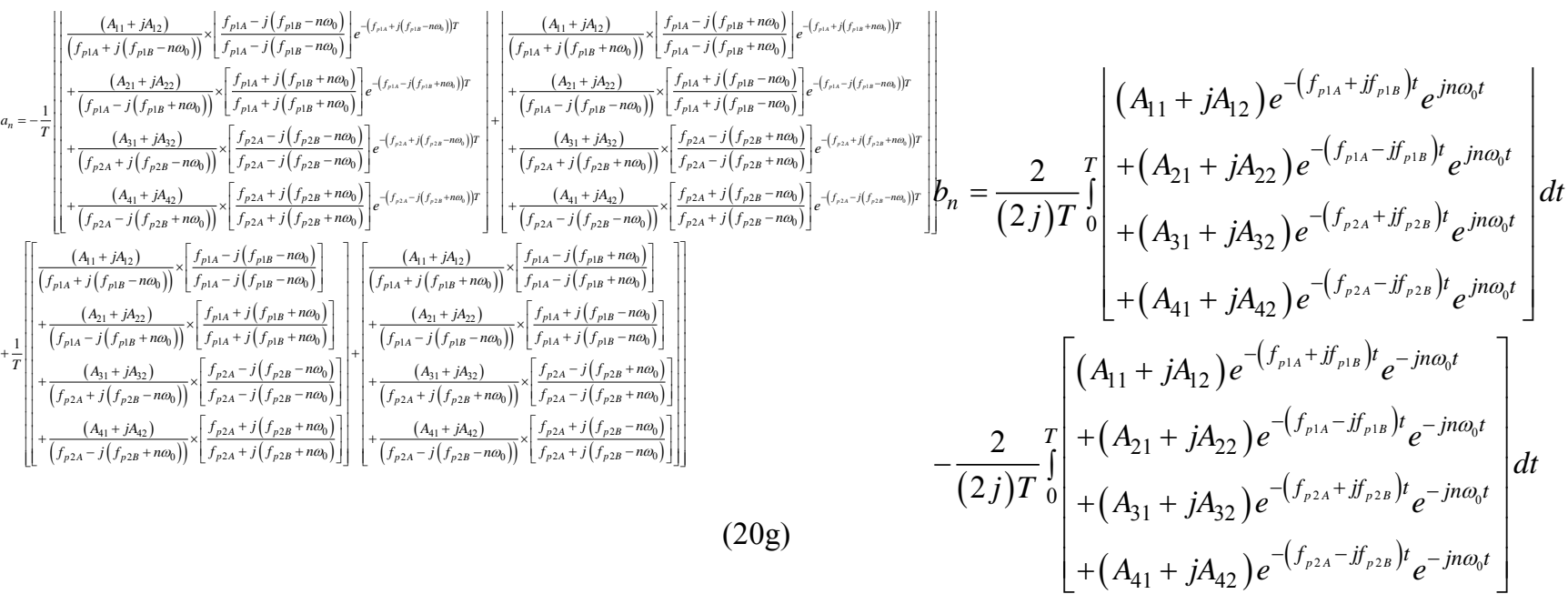

The Fourier coefficient can be performing integration easily by grouping of the positive and negative exponential of 
imaginary frequency as follows

$$
\begin{gathered}
b_{n}=\frac{-j}{T} \int_{0}^{T}\left[\begin{array}{l}
\left(A_{11}+j A_{12}\right) e^{-\left(f_{p 1 A}+j\left(f_{p 1 B}-n \omega_{0}\right)\right) t} \\
+\left(A_{21}+j A_{22}\right) e^{-\left(f_{p 1 A}-j\left(f_{p 1 B}+n \omega_{0}\right)\right) t} \\
+\left(A_{31}+j A_{32}\right) e^{-\left(f_{p 2 A}+j\left(f_{p 2 B}-n \omega_{0}\right)\right) t} \\
+\left(A_{41}+j A_{42}\right) e^{-\left(f_{p 2 A}-j\left(f_{p 2 B}+n \omega_{0}\right)\right) t}
\end{array}\right] d t \\
+\frac{j}{T} \int_{0}^{T}\left[\begin{array}{c}
\left(A_{11}+j A_{12}\right) e^{-\left(f_{p 1 A}+j\left(f_{p 1 B}+n \omega_{0}\right)\right) t} \\
+\left(A_{21}+j A_{22}\right) e^{-\left(f_{p 1 A}-j\left(f_{p 1 B}-n \omega_{0}\right)\right) t} \\
+\left(A_{31}+j A_{32}\right) e^{-\left(f_{p 2 A}+j\left(f_{p 2 B}+n \omega_{0}\right)\right) t} \\
+\left(A_{41}+j A_{42}\right) e^{-\left(f_{p 2 A}-j\left(f_{p 2 B}-n \omega_{0}\right)\right) t}
\end{array}\right] d t
\end{gathered}
$$

(21c)

The integration of exponential of complex frequency can be performed with equation (21c). Then, the limit of integration (which are the period and zero) can be seen at the right hand side of the bracket.

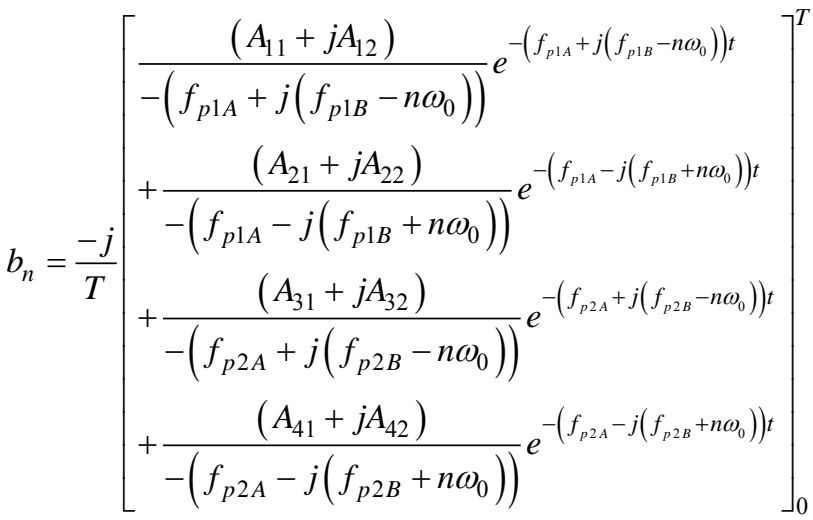

$$
\begin{aligned}
& +\frac{j}{T}\left[\begin{array}{c}
\frac{\left(A_{11}+j A_{12}\right)}{-\left(f_{p 1 A}+j\left(f_{p 1 B}+n \omega_{0}\right)\right)} e^{-\left(f_{p 1 A}+j\left(f_{p 1 B}+n \omega_{0}\right)\right) t} \\
+\frac{\left(A_{21}+j A_{22}\right)}{-\left(f_{p 1 A}-j\left(f_{p 1 B}-n \omega_{0}\right)\right)} e^{-\left(f_{p 1 A}-j\left(f_{p 1 B}-n \omega_{0}\right)\right) t} \\
+\frac{\left(A_{31}+j A_{32}\right)}{-\left(f_{p 2 A}+j\left(f_{p 2 B}+n \omega_{0}\right)\right)} e^{-\left(f_{p 2 A}+j\left(f_{p 2 B}+n \omega_{0}\right)\right) t} \\
+\left(f_{p 2 A}-j\left(f_{p 2 B}+j A_{42}\right)\right.
\end{array} e^{-\left(f_{p 2 A}-j\left(f_{p 2 B}-n \omega_{0}\right)\right) t}\right]_{0}^{T}
\end{aligned}
$$

The minus sign is the result of integration of exponential of complex frequency as a function of time. This minus sign can be move out of the bracket. Then, the limit of integration can be substitute into the time variable as follows

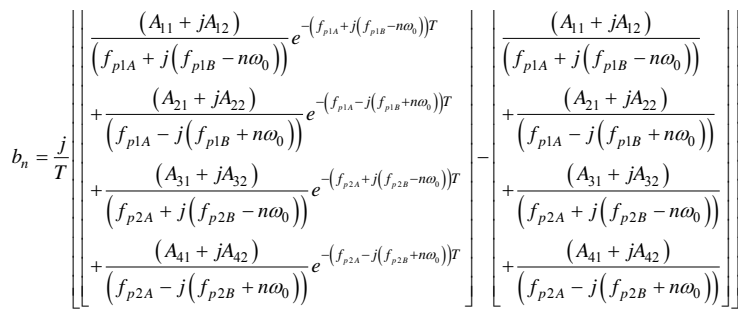

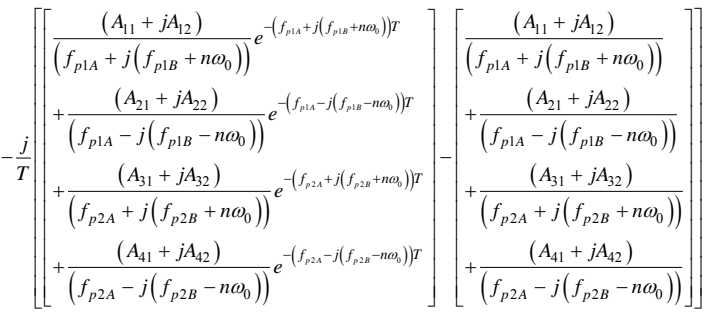

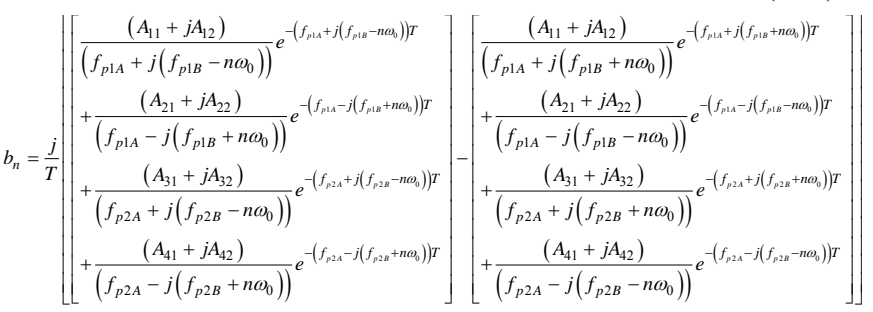

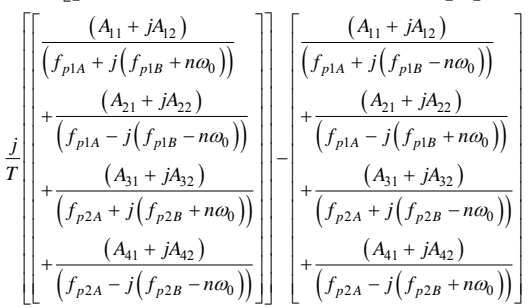

It is necessary to multiply each denominator with its complex conjugation to each term in equation (21f). As a result, it can be written as follow

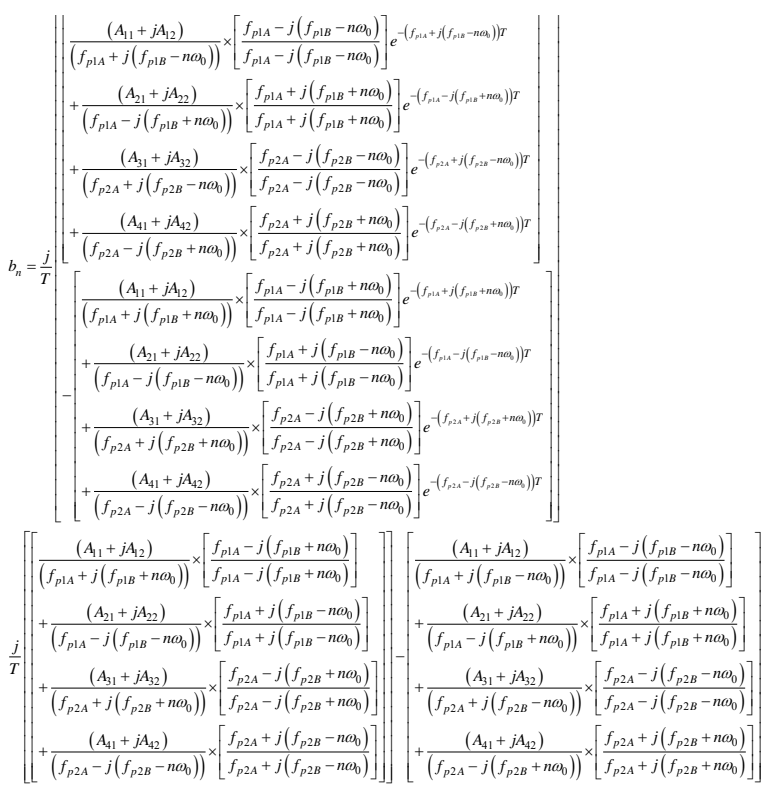

(21g) 


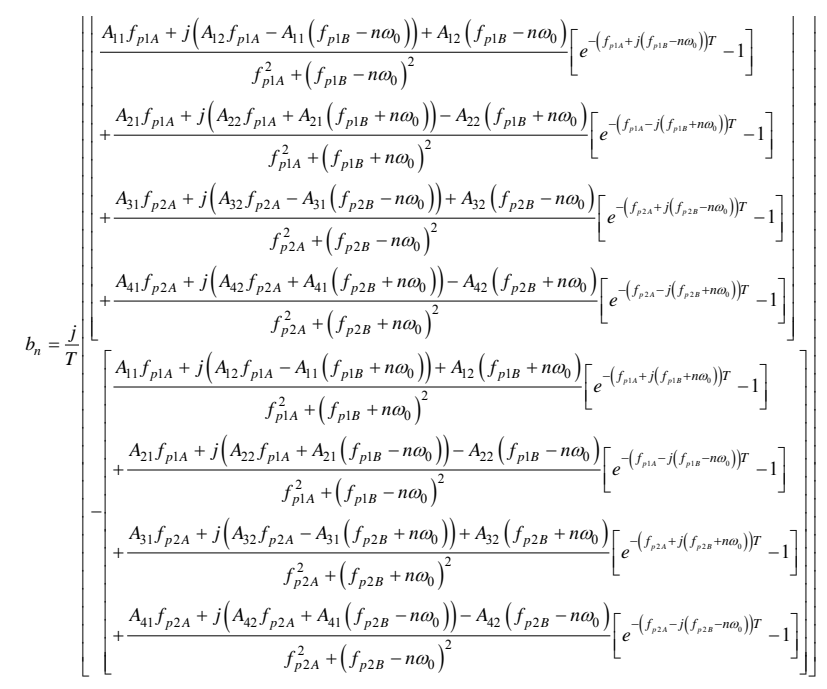

(21h)

$\left(C_{g s 2}+C_{d b 2}+C_{g d 1}+C_{g s 1}\right)\left(g_{d s 1}+\left(\frac{1}{R_{1}}\right)\right)=-\left(g_{m 2}+g_{m b 2}+g_{d s 2}\right)\left(\begin{array}{l}C_{d b 1}+C_{1}+C_{g s 2} \\ +C_{g d 2}+C_{g d 1}\end{array}\right)$

$\left(C_{d b 1}+C_{1}+C_{g s 2}+C_{g d 2}+C_{g d 1}\right)=-\frac{\left(C_{g s 2}+C_{d b 2}+C_{g d 1}+C_{g s 1}\right)\left(g_{d s 1}+\left(\frac{1}{R_{1}}\right)\right)}{\left(g_{m 2}+g_{m b 2}+g_{d s 2}\right)}$

$C_{1}=-\left(\frac{a_{2} a_{5}}{a_{3}}\right)-\left(C_{d b 1}+C_{g s 2}+C_{g d 2}+C_{g d 1}\right)$

\section{A DESIGN BY SIMULATION WITH MATLAB}

A design by simulation with MATLAB is performing by declare the levell drain current formula with 0.5 micron process parameters from textbook [4]. The small signal parameters such as transconductance, drain-source conductance and parasitic capacitances can be computed by MATLAB. Six Aspact ratio (width and length) of the transistors can be designed by assuming drain to source voltage and gate to source for each transistors. Usually, drain to source and gate to source voltage drop of the input transistor M3 and M6 have a half supply voltage drop. After all the physical constants are defined in MATLAB, the small signal parameters are computed. As a consequence, the coefficients in equation (5) can be determined as a numeric. For example, input impedance can be determined as

$$
\begin{aligned}
& Z_{\text {in }}=\left[\frac{\left(s^{3} b_{18}+s^{2} b_{19}+s b_{20}+b_{21}\right)}{\left(s^{4} b_{22}+s^{3} b_{23}+s^{2} b_{24}+s b_{25}+b_{26}\right)}\right] \\
& b_{18}=1.188 \times 10^{-44}, b_{19}=2.73 \times 10^{-33} \\
& b_{20}=2.605 \times 10^{-22}, b_{21}=7.026 \times 10^{-12} \\
& b_{22}=2.166 \times 10^{-59}, b_{23}=1.052 \times 10^{-36}, b_{24}=1.634 \times 10^{-36} \\
& b_{25}=1.44 \times 10^{-25}, b_{26}=5.679 \times 10^{-15} \\
& k_{n}=\mu_{n} C_{o x}=1.6698 \times 10^{-4} \\
& C_{1}=C_{2}=1 \times 10^{-15} \mathrm{~F}, R_{1}=R_{2}=11.66 \mathrm{k} \Omega
\end{aligned}
$$

Drain current of transistor M1, M3, M4 and M6 are designed to have the same current with 60 microampere. So that aspect ratio of these transistors will be above 1 (1.122). Drain current of transistor M2 and M5 are designed to have the same current with 220 microampere. As a result, aspect ratio of these transistors will be above 1 (1.029).

$$
\begin{aligned}
& Z_{i n}=\left[\frac{\left(s^{3} b_{18}+s^{2} b_{19}+s b_{20}+b_{21}\right)}{\left(s+f_{p 1}\right)\left(s+f_{p 2}\right)\left(s+f_{p 3}\right)\left(s+f_{p 4}\right)}\right] \\
& f_{p 1}=-4.8550 \times 10^{22} \\
& f_{p 2}=-1.7541 \times 10^{7} \\
& f_{p 3}=8.7705 \times 10^{6}+1.5195 \times 10^{7} i \\
& f_{p 4}=8.7705 \times 10^{6}-1.5195 \times 10^{7} i \\
& Z_{i n}= {\left[\frac{\left(s^{3} b_{18}+s^{2} b_{19}+s b_{20}+b_{21}\right)}{\left(s^{4} b_{22}+s^{3} b_{23}+s^{2} b_{24}+s b_{25}+b_{26}\right)}\right] } \\
& b_{18}=3.2151 \times 10^{-43}, b_{19}=5.286 \times 10^{-32}(23) \\
& b_{20}=1.2681 \times 10^{-21}, b_{21}=7.0258 \times 10^{-12} \\
& b_{22}=6.0495 \times 10^{-58}, b_{23}=2.0205 \times 10^{-35}, b_{24}=2.1085 \times 10^{-35} \\
& b_{25}=6.3840 \times 10^{-25}, b_{26}=5.679 \times 10^{-15} \\
& k_{n}=\mu_{n} C_{o x}=1.6698 \times 10^{-4} \\
& C_{1}=C_{2}=10 \times 10^{-15} F, R_{1}=R_{2}=11.66 \mathrm{k} \Omega \\
& Z_{i n}= \\
&\left.f_{p 1}=-3 \frac{\left(s^{3} b_{18}+s^{2} b_{19}+s b_{20}+b_{21}\right)}{\left(s+f_{p 1}\right)\left(s+f_{p 2}\right)\left(s+f_{p 3}\right)\left(s+f_{p 4}\right)}\right] \\
&\left.f_{p 2}=-6.5488 \times 10^{6}\right) \\
& f_{p 3}=3.2744 \times 10^{6}+5.6742 \times 10^{6} i \\
& f_{p 4}=3.2744 \times 10^{6}-5.6742 \times 10^{6} i
\end{aligned}
$$




$$
\begin{aligned}
& Z_{\text {in }}=\left[\frac{\left(s^{3} b_{18}+s^{2} b_{19}+s b_{20}+b_{21}\right)}{\left(s^{4} b_{22}+s^{3} b_{23}+s^{2} b_{24}+s b_{25}+b_{26}\right)}\right] \\
& b_{18}=2.5903 \times 10^{-37}, b_{19}=3.8563 \times 10^{-26} \\
& b_{20}=1.1197 \times 10^{-18}, b_{21}=7.0258 \times 10^{-12} \\
& b_{22}=4.9104 \times 10^{-52}, b_{23}=1.4865 \times 10^{-29}, b_{24}=1.26187 \times 10^{-29} \\
& b_{25}=5.49448 \times 10^{-22}, b_{26}=5.679 \times 10^{-15} \\
& k_{n}=\mu_{n} C_{o x}=1.6698 \times 10^{-4} \\
& C_{1}=C_{2}=10 \times 10^{-12} \mathrm{~F}, R_{1}=R_{2}=11.66 \mathrm{k} \Omega \\
& Z_{\text {in }}=\left[\frac{\left(s^{3} b_{18}+s^{2} b_{19}+s b_{20}+b_{21}\right)}{\left(s+f_{p 1}\right)\left(s+f_{p 2}\right)\left(s+f_{p 3}\right)\left(s+f_{p 4}\right)}\right] \\
& f_{p 1}=-3.027 \times 10^{22} \\
& f_{p 2}=-7.2390 \times 10^{4} \\
& f_{p 3}=3.6194 \times 10^{4}+6.2985 \times 10^{4} i \\
& f_{p 4}=3.6194 \times 10^{4}-6.2985 \times 10^{4} i \\
& Z_{\text {in }}=\left[\frac{\left(s^{3} b_{18}+s^{2} b_{19}+s b_{20}+b_{21}\right)}{\left(s^{4} b_{22}+s^{3} b_{23}+s^{2} b_{24}+s b_{25}+b_{26}\right)}\right] \\
& b_{18}=2.5897 \times 10^{-31}, b_{19}=3.8550 \times 10^{-20} \\
& b_{20}=1.1196 \times 10^{-15}, b_{21}=7.0258 \times 10^{-12} \\
& b_{22}=4.9093 \times 10^{-46}, b_{23}=1.4861 \times 10^{-23}, b_{24}=1.2611 \times 10^{-23} \\
& b_{25}=5.49359 \times 10^{-19}, b_{26}=5.6791 \times 10^{-15} \\
& k_{n}=\mu_{n} C_{o x}=1.6698 \times 10^{-4} \\
& C_{1}=C_{2}=10 \times 10^{-9} F, R_{1}=R_{2}=11.66 \mathrm{k} \Omega \\
& Z_{\text {in }}=\left[\frac{\left(s^{3} b_{18}+s^{2} b_{19}+s b_{20}+b_{21}\right)}{\left(s+f_{p 1}\right)\left(s+f_{p 2}\right)\left(s+f_{p 3}\right)\left(s+f_{p 4}\right)}\right] \\
& f_{p 1}=-3.027 \times 10^{22}, f_{p 2}=-7.0898 \times 10^{2} \\
& f_{p 3}=3.5406 \times 10^{2}+6.4316 \times 10^{2} i \\
& f_{p 4}=3.5406 \times 10^{2}-6.4316 \times 10^{2} i
\end{aligned}
$$

\section{CONCLUSION}

From simulation with MATLAB, the proposed circuit may not oscillate because real part of the pole could not minimize to zero by varying capacitor or drain current. The Fourier coefficient of the proposed circuit can be derived to have a complex amplitude and a complex form of trigonometric function. The impulse sensitivity function could be plotted from the derived equation. The future work may try to derive the input impedance of other type of active inductor with the hope that it may oscillate in the structure of common-source cross coupled with active inductor.

\section{References}

[1] B. Razavi, "RF Microelectronics" $2^{\text {nd }}$ edition, copyright 2012

[2] B. Razavi, "Design of Analog CMOS integrated circuit", McGrawHill, copyright 2000

[3] P.R Gray, R.G. Meyer, "Analysis and Design of Analog Integrated Circuit", John Wiley \& Sons, $4^{\text {th }}$ edition, copyright 2001

[4] A.S Sedra, K.C Smith, "Microelectronic Circuit", Oxford University Press, $7^{\text {th }}$ edition, copyright 2015.

[5] S. Hara, T. Tokumitsu, "Monolithic Microwave Active Inductors and their Applications", pp. 1857-1860

[6] S. Hara, T. Tokumitsu, M. Aikawa, "Lossless Broadband Monolithic Microwave Active Inductors" IEEE Transactions on Microwave Theory and Techniques, Vol.37, No.12, December 1989, pp. 1979-1984

[7] H. Hagiwara, M. Kumazawa, S. Takagi, M. Furihata, M. Furihata, M. Nagata, T. Yanagisawa, "A Monolithic Video Frequency Filter using NIC-based Gyrators", IEEE Journal of Solid-State Circuits, Vol.23, No.1, February 1988, pp. 175-182

[8] S. El Khoury, "The Design of Active Floating Positive and Negative Inductors in MMIC Technology", IEEE Microwave and Guided Wave Letters, Vol.5, No.10, October 1995, pp. 321-323

[9] R. Duncan, K. W. Martin, A. S. Sedra, “A Q Enhanced Active RLC Bandpass Filter", IEEE Transactions on Circuits and Systems II, Analog and Digital Signal Processing, Vol.44, No.5, May 1997, pp. 341-347

[10] D. Shmilovitz, I. Yaron, S. Singer, "Transmission Line Base Gyrator", IEEE Transactions on Circuits and Systems I: Fundamental Theory and Applications, Vol.45, No.4, April 1998, pp. 428-433

[11] U. Yodprasit, J. Ngarmnil, "Q-Enhancing Technique for RF CMOS Active Inductor", ISCAS2000, pp. V589-V-592

[12] A. Thanachayanont, "Low Voltage CMOS Fully Differential Active Inductor and Its application to RF Bandpass Amplifier Design”, pp. 125-128

[13] A. Thanachayanont, "CMOS transistor only active Inductor for IF/RF applications”, ICIT'02, 1209-1212

[14] T. Adachi, S. Izumiya, "A MOS High Q Active Inductor, Principle and Fundamental Characteristics", pp. $833-835$

[15] R. Y. Barazarte, G. G. Gonzalez, M. Ehsani, "Generalized Gyrator Theory", IEEE Transactions on Power Electronics, Vol.25, No.7, July 2010, pp. 18321837

[16] R. Mehra, V. Kumar, A. Islam, "Reliable and Q Enhanced Floating Active Inductors and their application in RF Bandpass Filters", IEEE Access, Vol.6, 2018, pp. 48181-48194

[17] K. Tripetch, N. Nakano, "A Design of Cross-Coupled Oscillator with approximate $4^{\text {th }}$ order polynomial rooting formula", WSEAS Transactions on Electronics" Vol.9, 2018, pp.79-84 
[18] K. Tripetch, N. Nakano, "A Design of Cross-Coupled Oscillator with Exact $4^{\text {th }}$ order polynomial rooting formula", AIP Conference Proceedings 1982, 020046 ( 2018); http://doi.org/10.1063/1.5045452

[19] K. Tripetch, N. Nakano, "A Design of Cross Couple Oscillator with Root Locus by using Current Consumption", J. Electrical Engineering \& Electronic Technology, Volume7, Issue2, 1000158, pp. 1-6, DOI: $10.4172 / 2325-9833$

[20] A. Hajimari, T. H. Lee, "The Design of Low Noise Oscillators", Kluwer Academic Publishers, copyright 1999

[21] William H. Hayt, Jack E. Kemmerly, Steven M Durbin, "Engineering Circuit Analysis", $8^{\text {th }}$ edition, copyright 2012, pp. 738-751

[22] M. P. Sarma, K. K. Sarma, “A Transmission gate based High frequency rectifier designed using $45 \mathrm{~nm}$ CMOS Process for RF Energy Harvesting Application, WSEAS Transactions on Circuits and Systems, Volume18, 2019, pp. $44-49$

[23] H. Akhamal, M. Chakir, H. Ameziane, M. Akhamal, K. Zared, H. Qjidaa, “ Nano-power Low-dropout voltage Regulator Circuit in $90 \mathrm{~nm}$ CMOS Technology for RF SoC Applications, WSEAS Transactions on Power Systems, Volume 15, 2020, pp. 240-248

[24] H. Akhamal, M. Chakir, H. Ameziane, M. Akhamal, Z. Zared, H. Qjidaa, "A $916 \mathrm{nW}$ power LDO Regulator Circuit in 90-nm CMOS Technology for RF SoC Applications, WSEAS Transactions on Circuits and Systems, Volume 19, 2020, pp. 311-319

Kittipong Tripetch (M'03,GSM'14,M'19) was born in Bangkok since 15 September 1972. He received his Bachelor and Master degree in Telecommunication Engineering and Microelectronic Engineering since 1996,2002, respectively from Mahanakorn University of Technology. He have been teaching electronic circuit and control system at a division of electronic and telecommunication engineering, Faculty of Engineering and Architecture at Rajamangala University of Technology Suvarnabhumi since August 2004. Since April 2014, he have been studying Ph.D degree in integrated design engineering at Keio University where he received his Ph.D degree in March 2019. He came back to teach electronic and control systems further since February 2018.

He wrote a book title "Engineering Electronics" in Thai language which was published by Chulalongkorn University Press since 2015. He published his research papers in IEEE Explore 9 papers since 2020.

He received an IEEE member status since 2003. After he had been writing his Ph.D thesis since April 2014 he received an IEEE Graduate member status until he received member status again since 2019 after he received his Ph.D degree from Keio University since the end of March 2019.

\section{Contribution of Individual Authors to the Creation of a Scientific Article (Ghostwriting Policy)}

Parkpoom Khanthawiworn carried out the simulation and the optimization of MATLAB pole position

Kittipong Tripetch carried out the writing idea, circuit diagram description, derivation of the design formulas.

\section{Creative Commons Attribution License 4.0 (Attribution 4.0 International, CC BY 4.0)}

This article is published under the terms of the Creative Commons Attribution License 4.0

https://creativecommons.org/licenses/by/4.0/deed.en_US 